\title{
Physics with diproton final states at ANKE-COSY
}

\author{
S. Dymov ${ }^{1,2, a}$ for the ANKE collaboration \\ 1 Physikalisches Institut II, Universität Erlangen-Nürnberg, 91058 Erlangen, Germany \\ 2 Laboratory of Nuclear Problems, Joint Institute for Nuclear Research, 141980 Dubna, Russia
}

\begin{abstract}
The study of processes with production of proton pairs with small excitation energy (diprotons) provides a new approach to hadron interactions at intermediate energies. It is especially instructive due to comparison with processes where a deuteron is produced in the same kinematics. Provided that the momentum transfer is high enough the diprotons are found presumably in the ${ }^{1} S_{0}$ state, and this simplifies the theoretical analysis. The ANKE spectrometer provides the possibility to select the diprotons with sufficiently high excitation energy resolution. A number of processes including $p d \rightarrow\{p p\}_{s} n$, production of one and two pions in $p N$ collisions, and the $p p \rightarrow\{p p\}_{s} \gamma$ process, have been investigated.
\end{abstract}

\section{Experiments with diproton final states at ANKE}

Few nucleon systems have long been a subject of intensive theoretical and experimental study. A particularly important role in this study is played by processes with production of the deuteron, a bound state of proton and neutron. In this respect, a particular interest arises for the investigation of the diproton, an unbound system of two protons with small excitation energy. The diproton, being the isospin partner of the deuteron, possesses different quantum numbers. Selection of the excitation energy of the proton pair $E_{p p}<3 \mathrm{MeV}$ ensures the dominance of the ${ }^{1} S_{0}$ state of the diproton, which simplifies significantly the theoretical interpretation. The reactions with formation of a diproton involve transitions in the NN system, different from the case of the deuteron, and, in particular, the role of the $\Delta$ isobar is expected to be much suppressed because the $\mathrm{S}$-wave $\Delta N$ intermediate state is forbidden.

Detection of the proton pair with $E_{p p}<3 \mathrm{MeV}$ in an experiment requires an excellent excitation energy resolution and the ability to resolve the two spatially close trajectories of the protons. The ANKE spectometer [1] provides a resolution of $\sigma\left(E_{p p}\right)=0.2-0.6 \mathrm{MeV}$ in the $E_{p p}=0-3$ $\mathrm{MeV}$ range and is particularly well suited for this purpose.

The diproton program at ANKE involves several experiments:

- Study of meson-less deuteron break-up $p d \rightarrow\{p p\}_{s} n$ in collinear kinematics:

- at high momentum transfer [2-4],

- at low momentum transfer (charge-exchange reaction) [5].

- Study of meson production in $p N \rightarrow\{p p\}_{S} X$, where $X$ is:

- a single pion [6],

a e-mail: s.dymov@fz-juelich.de
- the $(\pi \pi)$ system, used to study of the ABC effect in $p p$ collisions [7],

- a heavier meson like $\eta$ and $\omega$.

- Inverse diproton photodisintegration $p p \rightarrow\{p p\}_{s} \gamma$. [8].

It should be mentioned that investigation of single pion production and the charge-exchange reaction are the key double polarization experiments in the future physics program at ANKE.

\section{Deuteron break-up reactions}

\subsection{High momentum transfer}

The process to be studied is $p d \rightarrow\{p p\}_{s} n$ with a forward emitted diproton. The kinematics of the process is the same as in $p d$-backward scattering and the same mechanisms can be considered for its description. In contrast to $p d \rightarrow d p$, the amplitude of excitation of $\Delta$ in the intermediate state is expected to be suppressed by a factor of 3 due to selection of the diproton in an $S$-wave. Thus, the one nucleon exchange mechanism, which is sensitive to the short range part of the $\mathrm{NN}$-interaction potential and to the high momentum part of the deuteron wave function, is expected to play a more important role.

The differential cross-section of $p d \rightarrow\{p p\}_{s} n$ with a forward emitted diproton has been measured in the range of $T_{p}=0.5-2.0 \mathrm{GeV}$ [2]. Comparison of the results with a model-calculation including $\Delta$-production, one nucleon exchange and single scattering mechanisms, shows a clear preference in using the modern CD Bonn NN-potential over the Paris and RSC potentials in the region $0.4-0.6$ $\mathrm{GeV} / \mathrm{c}$ for the internal deuteron momentum [2]. Preliminary results of a new high statistics measurement of the cross section [4] are presented in Fig. 1. The increased statistics allows one to obtain angular distributions and to test whether the diproton is indeed found in the ${ }^{1} S_{0}$ state. 


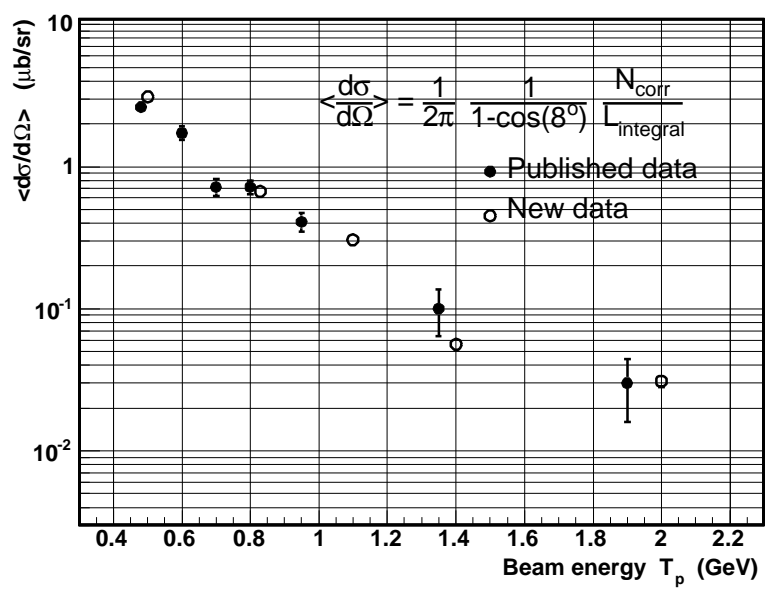

Fig. 1. Forward differential cross section of the $p d \rightarrow\{p p\}_{s} n$ process averaged in the range $\cos \theta_{p p}=(0-8)^{o}$.

For further insight, additional data, in particular polarization measurements, are needed to provide a complete set of observables.

\subsection{Deuteron charge-exchange break-up reaction}

The processes $\mathbf{d p} \rightarrow\{p p\}_{s} n$ with a forward emitted diproton proceed with low momentum transfer. One can extract the elementary charge-exchange spin amplitudes of $p n$ scattering from the measured polarization observables in the deuteron charge-exchange break-up process. The existing information on these amplitudes above $800 \mathrm{MeV}$ is scarce. The equivalent neutron beam momentum available for COSY ranges up to $1.1 \mathrm{GeV}$ in the case of a deuteron beam, and up to $2.8 \mathrm{GeV}$ in the case of a deuteron target.

As a first step of the study, a measurement at $T_{n}=585$ $\mathrm{MeV}$ was performed, where information on the np spindependent amplitudes is available. The analysis showed excellent agreement of the extracted $\mathrm{np}$ amplitudes with the existing data [5]. Currently, the data obtained at $T_{d}=$ $2.3 \mathrm{GeV}$ are being analyzed. New double polarization measurements of the spin correlation coefficients $C_{y, y}, C_{x, x}$ have been conducted at the end of 2009.

\section{Pion production in $p N \rightarrow\{p p\}_{s} \pi$}

\section{$3.1 \pi^{0}$ production in $0.5-2.0 \mathrm{GeV}$ region}

Single pion production in nucleon-nucleon collisions, $N N \rightarrow$ $N N \pi^{0}$, is one of the principial tools used in the investigation of NN dynamics at intermediate energies. Because of the large momentum transfers involved, even close to threshold, such a meson production is sensitive to the shortrange part of the $\mathrm{NN}$-interaction. The $p p \rightarrow(p p)_{s} \pi^{0}$ differential cross section has been measured with the ANKE spectrometer at COSY-Jülich for seven proton beam energies $T_{p}$ between 0.5 and $2.0 \mathrm{GeV}$ [6]. In the measured region of $\theta_{p p}^{c m}= \pm 18^{\circ}$, the data reveal a forward dip for $T_{p} \leq 1.4 \mathrm{GeV}$ whereas a forward peaking is observed at
$1.97 \mathrm{GeV}$. The energy dependence of the forward cross section shows a broad peak in the $0.6-0.8 \mathrm{GeV}$ region, probably associated with $\Delta(1232)$ excitation, and a minimum at $1.4 \mathrm{GeV}$.

The ratio of $p p \rightarrow(p p)_{s}\left(0^{\circ}\right) \pi^{0}$ to $p p \rightarrow d\left(0^{\circ}\right) \pi^{+}$cross sections can provide information on the relative strength of spin-singlet to spin-triplet production. The resulting ratio shows that singlet production in the forward direction remains small at energies even above the $\Delta(1232)$ excitation region. Below $1.4 \mathrm{GeV}$ the ratio is typically a few per cent, which is significantly smaller than the trivial spin-statistics factor of $1 / 3$.

\subsection{Near threshold region}

The spin structure of the process $p n \rightarrow\{p p\}_{s} \pi^{-}$(or $p p \rightarrow$ $\left.\{p p\}_{S} \pi^{0}\right)$ with the diproton in the ${ }^{1} S_{0}$ state is $\frac{1}{2}^{+} \frac{1}{2}^{+} \rightarrow$ $0^{+} 0^{+}$, and there are only two spin amplitudes involved in this process. From this it follows that the measurement of the differential cross section, the analyzing power and one spin correlation coefficient is sufficient to extract the magnitudes of the two amplitudes and their relative phase. A combined study of several observables in $p p \rightarrow\{p p\}_{s} \pi^{0}$ and $p n \rightarrow\{p p\}_{s} \pi^{-}$can be used to isolate the important strength parameter $d$ of the four-nucleon-pion contact interaction in $\chi \mathrm{PT}[9]$.

\subsection{New measurements}

In October 2007, the unpolarized cross section of the $p p \rightarrow$ $(p p)_{s}\left(0^{\circ}\right) \pi^{0}$ process has been measured at 6 new beam energies: $353,500,550,700,1700$ and $2400 \mathrm{GeV}$. These data will allow one to study the cross section energy dependence in the extended range and with a higher statistics.

In 2009 , the vector analysing power in the process $\mathbf{p} p \rightarrow(p p)_{s}\left(0^{\circ}\right) \pi^{0}$ has been obtained at 353, 500, 550 and $700 \mathrm{MeV}$, and a high precision measurement of the $\mathbf{p} n \rightarrow(p p)_{s}\left(0^{\circ}\right) \pi^{-}$process has been preformed. The results at $353 \mathrm{MeV}$ will be used for defining the four-nucleon-pion contact term in $\chi$ PT.

In addition, a double-polarized experiment for obtaining of $A_{y, y}$ and $A_{x, x}$ in $\mathbf{p} n \rightarrow(p p)_{s}\left(0^{\circ}\right) \pi^{-}$at $353 \mathrm{MeV}$ is planned [10].

\section{Inverse diproton photodisintegration}

The fundamental reaction $p p \rightarrow\{p p\}_{s} \gamma$ has been observed using the missing-mass technique at proton beam energies of $T_{p}=0.353,0.5$, and $0.55 \mathrm{GeV}$ [8]. This is equivalent to photodisintegration of a free ${ }^{1} S_{0}$ diproton for photon energies $E_{\gamma} \approx T_{p} / 2$.

The differential cross sections measured for c.m. angles $\theta_{p p}^{c m}<20^{\circ}$ exhibit a steep increase with angle that is compatible with E1 and E2 multipole contributions. The ratio of the measured cross sections to those of $n p \rightarrow \gamma d$ is on the $10^{-3}-10^{-2}$ level. The increase of the $p p \rightarrow\{p p\}_{s} \gamma$ 
cross section with $T_{p}$ might reflect the influence of the $\Delta(1232)$ excitation.

In addition, the analysis of data obtained at beam energies 625,700 and $800 \mathrm{MeV}$ reveals a signal of the $p p \rightarrow$ $\{p p\}_{s} \gamma$ process on a rather large background from $p p \rightarrow$ $\{p p\}_{s} \pi^{0}$. This result together with the published data at $T_{p}=353,500$, and $550 \mathrm{MeV}$ allows one to investigate the behavior of the forward cross section of diproton photodisintegration in the $\Delta$-isobar excitation region.

\section{$5 \mathrm{ABC}$ effect in pp collisions}

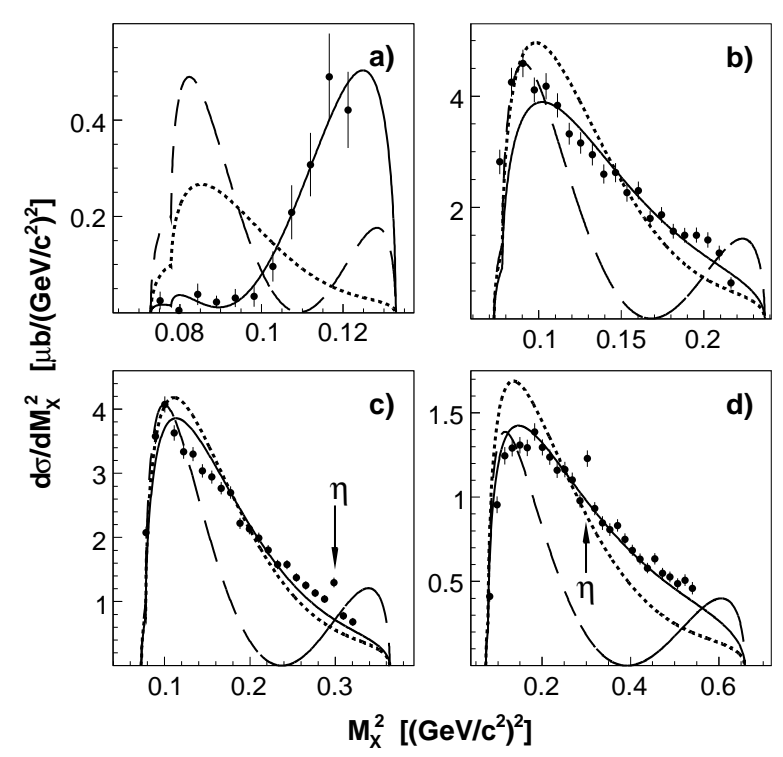

Fig. 2. The $p p \rightarrow p p X$ differential cross section as a function of the square of the missing mass $M_{x}$ at (a) 0.8 , (b) 1.1, (c) 1.4, and (d) $2.0 \mathrm{GeV}$ for $E_{p p}<3 \mathrm{MeV}$ and $\cos _{p p}>0.95$. The $\eta$ peaks are indicated. The curves correspond to the double $\Delta$ model [7] with the initial pp system in the pure S-wave $A_{D}=0$ (long dashes), pure D-wave $A_{S}=0$ (short-dashed), and with the best fit of $A_{S} / A_{D}$ ratio (solid line).

The cross section for inclusive multipion production in the $p p \rightarrow p p X$ reaction was measured at four beam energies, $0.8,1.1,1.4$, and $2.0 \mathrm{GeV}$, again for low excitation energy in the final pp system [7]. At the three higher energies, the missing-mass $M_{x}$ spectra show a strong enhancement at low $M_{x}$, corresponding to the Abashian-Booth-Crowe (ABC) effect that moves steadily to larger values as the energy is increased (see Fig. 2). Despite the missing-mass structure looking very different at $0.8 \mathrm{GeV}$, the variation with $M_{x}$ and beam energy are consistent with two-pion production being mediated through the excitation of two $\Delta$ (1232) isobars, coupled to $\mathrm{S}$ and $\mathrm{D}$ states of the initial $\mathrm{pp}$ system. There is no sign of any resonancelike structure in the energy dependence of the type recently observed for the $p n \rightarrow d \pi^{0} \pi^{0}$ total cross section [12].

\section{Acknowledgments}

This work is supported by JCHP-FFE program of FZ-Jülich.

\section{Bibliography}

\section{References}

1. S. Barsov et al., NIM A 462 (2001) 364

2. V. Komarov et al., Phys. Lett. B 553 (2003) 179

3. S. Yaschenko et al., Phys. Rev. Lett. 94 (2005) 072304

4. S. Dymov et al., submitted to Phys. Rev. C (2009)

5. D. Chiladze et al., Phys. Lett. B 637 (2006) 170

6. V. Kurbatov et al., Phys. Lett. B 661 (2008) 22

7. S. Dymov et al., Phys. Rev. Lett. 102 (2009) 192301

8. V. Komarov et al., Phys. Rev. Lett. 101 (2008) 102501

9. C. Hanhart, Phys. Rep. 397 (2004) 155

10. Spin Physics From COSY To FAIR, COSY proposal \# 152, nucl-ex/0511028

11. COSY proposal \# 192, http://www.fzjuelich.de/ikp/anke/en/proposals.shtml

12. M. Bashkanov et al., Phys. Rev. Lett. 102 (2009) 052301 